\title{
A NONLINEAR MODEL FOR VASOCONSTRICTION
}

\author{
John Ringwood* Violeta Mangourova* \\ Sarah-Jane Guild ${ }^{* *}$ Simon Malpas ${ }^{* *}$
}

* Dept. of Electronic Eng., NUI Maynooth, Ireland

** Dept. of Physiology, Uni. of Auckland, New Zealand

\begin{abstract}
The control of blood pressure is a complex mixture of neural, hormonal and intrinsic interactions at the level of the heart, kidney and blood vessels. While experimental approaches to understanding these interactions remain useful, it remains difficult to conduct experiments to quantify these interactions as the number of parameters increases. Thus modelling approaches can offer considerable assistance. Typical mathematical models which describe the ability of the blood vessels to change their diameter (vasoconstriction) assume linearity of operation. However, due to the interaction of multiple vasocontrictive and vasodilative effectors, there is a significant nonlinear response to the influence of neural factors, particularly at higher levels of nerve activity (often seen in subjects with high blood pressure) which leads to low blood flow rates. This paper proposes a nonlinear mathematical model for the relationship between neural influences (sympathetic nerve activity (SNA) and blood flow, using a feedback path to model the predominently nonlinear effect of local vasoactive modulators such as Nitric Oxide, which oppose the action of SNA. The model, the structure of which is motivated by basic physiological principles, is parameterised using a numerical optimisation method using open-loop data collected from rabbits. The model responses are shown to be in good agreement with the experimental data. Copyright C 2006 IFAC.
\end{abstract}

Keywords: Biomedical systems, mathematical model, blood flow, nonlinear analysis, numerical optimisation.

\section{INTRODUCTION}

The regulation of blood pressure is critical in maintaining nutrient and oxygen supply to the various perfused organs. Blood pressure is determined according to the (Ohm's Law) relationship:

$$
M A P=C O . T P R
$$

where:

MAP is the mean (of the systolic and diastolic) pressure (measured in $\mathrm{mmHg}$ ),
CO is cardiac output, evaluated as the product of heart rate and stroke volume (in l/s), and

TPR is the total peripheral resistance as seen by the heart (in $\mathrm{mmHg} \mathrm{s} / \mathrm{l}$ ).

This study will focus on those components which mediate the resistance to blood flow, with the primary pump, the heart, assumed to have a relatively constant output. Blood flow may be differentially regulated according to physiological needs at any particular time via a variety of hormonal, neural and intrinsic factors. 
In particular, the emphasis is on TPR and how it mediates blood flow/pressure on a relatively short timescale i.e. seconds. Central to this timescale, with a time delay between stimulation and response of 0.6s (Guild et al., 2001), is the neural control of blood pressure, with sympathetic innervation of a number of major organs and areas of the vasculature, allowing rapid control of resistance via the central nervous system. Such sympathetic nerve activity (SNA) causes the release of neurotransmitters which, in general, cause the smooth muscle surrounding small arteries and arterioles to constrict (DiBona and Kopp, 1997).

The distribution of sympathetic innervation throughout the vasculature (and the nature of the local receptors i.e. whether they cause vasoconstriction or vasodilation) determines the action that will take place at any particular site. However, in addition to neural control, several other mechanisms have significant effect on resistance, including:

- Hormones, which circulate throughout the system and can effect both vasodilation or vasoconstriction, depending on the particular hormone and the type of receptor it binds to (typical hormones include Epinephrine, Antidiuretic Hormone, Angiotensin II and Cortisol (Sorensen et al., 2000; Bellomo et al., 1999)),

- Intrinsic factors (myogenic autoregulation), which regulate blood vessel compliance and, for example, produce a vasoconstrictive action in the smooth muscle in response to a distorting force on the walls of blood vessels due to blood pressure (Navar, 1998),

- Paracrines, which are humoral substances that are secreted by cells in the endothelium (the area between the circulating blood and the vascular smooth muscle) and affect neighboring cells. Paracrines can have both vasodilatory (e.g. Prostacyclin, Nitric Oxide) and vasoconstrictive effects (e.g. Thromboxane, Endothelin-1), with some paracrines having both dilatory and constrictive potential (e.g. Endothelin-1 (Boulanger and Vanhoutte, 1998)), depending on the receptors they bind to.

- Metabolic factors, which can elicit vasoaction in response to local metabolic demands. Typical mediators include oxygen (constriction).

Fig.1 (adapted from (Richardson et al., 1998)) attempts to summarise the various factors involved in mediating vasoaction. The vasoactive mechanisms can be loosely grouped into systemic effects (e.g. SNA, hormones) and local effects (paracrines, tissue metabolites). The vasoaction at any particular site is therefore likely to be a combination of both factors, dependent on:

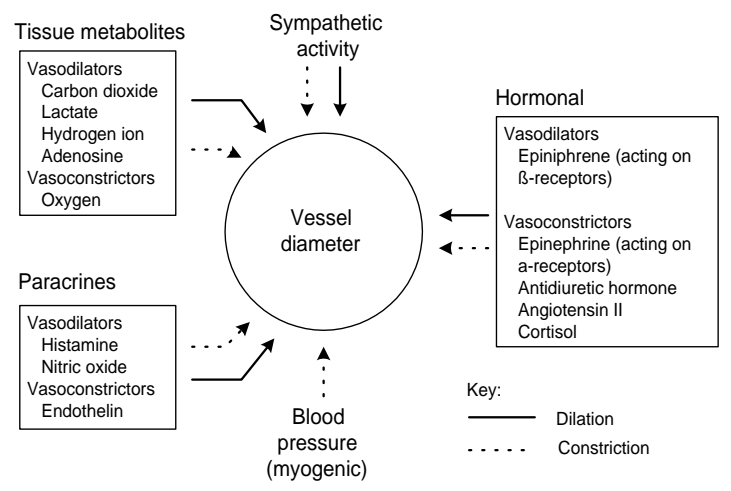

Fig. 1. Summary of vasoactive mechanisms

- The systemic requirements (mainly regulation of blood pressure), and

- Local requirements, including metabolic needs.

The study in this paper examines the renal vasculature in particular and attempts to build a mathematical model relating blood flow to SNA, with hormones, paracrines, etc. as mediating factors.

Mathematical models can be used to develop understanding of the system under study if the structure, and parameters, of the model can be validated by good agreement between model output and experimental data. In order to achieve this structural information, the emphasis should be on models which attempt to exploit the physical system description, rather than adopting a 'bulk' black box modelling approach. While the latter approach can give a very good model fit for specific experimental data, it does little to reveal the generic structure of the system under study.

To date, a number of attempts have been made to model the blood flow response to SNA. However, most techniques focus on linear models, which fail to capture essential aspects of the response. For example, the paper by Eppel et al. (2003) considers only broad magnitude changes in renal blood flow (RBF) in response to SNA stimulation, while the paper by Leonard et al. (2000) fits an unparameterised frequency response to the RBF/SNA relationship. Navakatikyan et al. (2000) fit a first-order (pole-only) model to the response, with Guild et al. (2001) using a frequency-domain approach to fit a linear $4^{\text {th }}$ order (4 poles and 2 zeros plus delay) model to the data. While the linear model of Guild et al. (2001) gives a good fit at relatively low SNA amplitudes, the response match deteriorates as higher SNA stimulation evokes reactionary responses in (local) vasodilatory mechanisms.

\section{MODEL DEVELOPMENT}

The essential model structure is shown in Fig.2. The neural control of the renal vasculature is considered central to the model for two reasons: 
(1) It is the most significant (and 'independent') input to the model i.e. most other mechanisms are considered reactionary on a more local level, and

(2) The experimental data on which the model will be validated is 'open-loop' as far as neural control of the vasculature is concerned. This is achieved via transection of the renal sympathetic nerve and artificial (electrical) stimulation of the renal nerves using a suitable excitation signal.

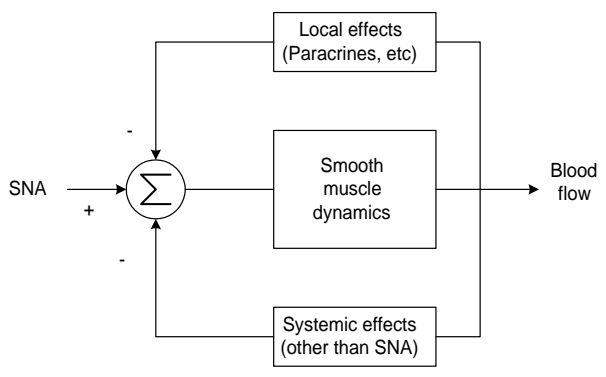

Fig. 2. Feedforward/feedback configuration

The two feedback paths represent the effects that both local and systemic blood pressure/flow control mechanisms have in response to SNA-based activation of renal blood flow. Since the renal vasculature is just one component which regulates blood pressure, the response from systemic mechanisms is unlikely to be nearly as significant as the response from local mechanisms. Therefore, a single feedback block will be employed in the structure as shown in Fig.2. This single block could incorporate systemic effects, but is configured to mainly model local effects. The model structure is based on the following physiological premise. Above a certain (threshold) value of (normal) blood flow, the response of blood flow to SNA is relatively linear. However, when blood flow drops below a certain value, local myogenic factors and paracrines work progressively harder (as blood flow decreases) to maintain an acceptable level of local blood flow. This combination of threshold and progressive response is captured by the 'activation level' block in Fig.3. The 'local dynamics' block in Fig.3 captures the speed of response of these local reactionary mechanisms. Finally, the 'smooth' muscle dynamics' block represents the dynamic response of the smooth muscle to a stimulus from an appropriate receptor.

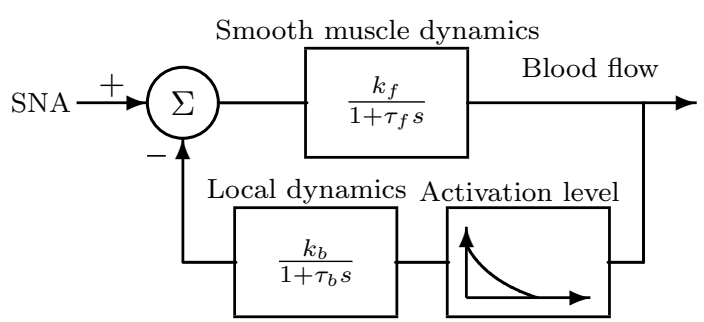

Fig. 3. Block diagram of proposed model structure

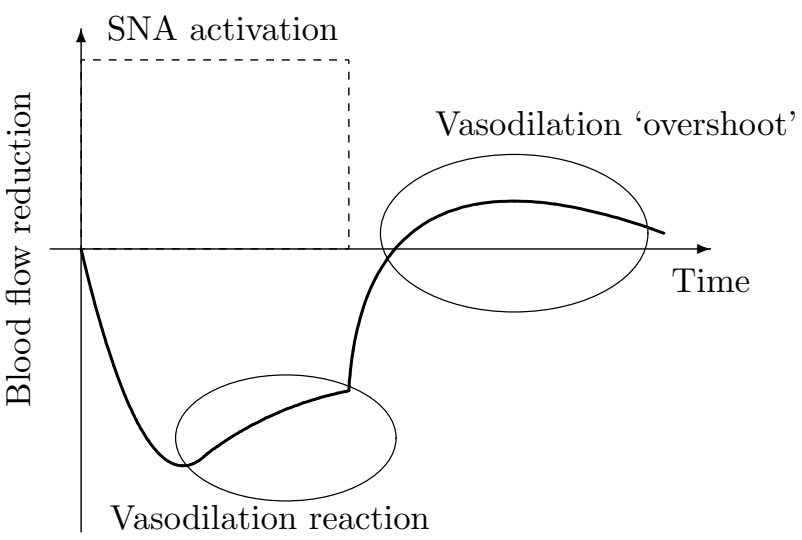

Fig. 4. Typical 'large-signal' response to SNA activation

Fig. 4 shows the typical type of response obtained from the model. The initial response to a step activation in SNA is roughly first order exponential, but as soon as blood flow reduction reaches a certain level, local (opposite) effects temper the response dramatically. Following release of the SNA activation, the response returns rapidly to the original level, assisted by the local paracrines, etc. which are still active and have not yet been dispersed. Finally, the blood flow overshoots its original value, due to the slow dispersal of these local vasodilatory effects.

From the above description, some aspects of the model can be clarified:

- The local vasodilatory response is not linear and has some 'threshold' of blood flow change above which it is activated,

- The response of the local vasodilatory reaction is significantly slower than that of the smooth muscle to the SNA stimulus (i.e. $\tau_{b}>\tau_{f}$ ), and

- The magnitude of the action (to SNA) and reaction (by the local vasodilatory mechanism) is comparable, at least to an order of magnitude.

\section{DATA AVAILABILITY}

Experiments were performed on 6 anaesthetized New Zealand white rabbits (Leonard et al., 2000). A transit time flow probe (type 2SB; Transonic Systems, Ithaca, NY, USA), connected to a compatible flowmeter (T106, Transonic Systems) was used to measure RBF, with arterial pressure being monitored using a catheter inserted into the central ear artery and connected to a pressure transducer (Cobe, Arvarda, CO). The measured signals were sampled at $500 \mathrm{~Hz}$, digitized and saved continuously as $2 \mathrm{~s}$ averages of each variable. In 
Table 1. Dynamic block parameters

\begin{tabular}{|c|c|c|c|}
\hline$k_{f}$ & $k_{b}$ & $\tau_{f}$ (secs) & $\tau_{b}$ (secs) \\
\hline 1 & 0.85 & 20 & 333 \\
\hline
\end{tabular}

addition, heart rate (HR, beats/min) was derived from the MAP waveform.

For stimulation, the renal nerves were placed across a pair of hooked stimulating electrodes and then sectioned proximal to the electrodes. Stimulation sequences using both amplitude (AM) and frequency modulation (FM) were applied, all using a pulse width of $2 \mathrm{mS}$. In the $\mathrm{AM}$ sequence, voltages of $0.5,1.0,1.5,2.0,3.0,5.0$ and $8.0 \mathrm{~V}$ were applied in random order at a constant frequency of $5 \mathrm{~Hz}$. For the FM sequence, frequencies of 0.5 , 1.0, 1.5, 2.0, 3.0, 5.0 and $8.0 \mathrm{~Hz}$ were applied in random order using a voltage equal to that required to produce a maximal RBF response. For both AM and FM sequences, the stimulation interval was 3 min., with a 5 min. recovery period before delivering the next stimulus.

\section{MODEL PARAMETERISATION}

Given the intuitive nature of the model and the strong relationship with the underlying physiology, initial attempts focussed on tuning the model parameters by trial and error. One further component was added to the model of Fig.3 in order to correctly represent the relationship between the varying levels of steady state response to the frequency of SNA stimulation. This (mildly) nonlinear characteristic preceeds the model section shown in Fig. 3 and is given by the transformation in Fig.5. The parameters of the dynamic feedfor-

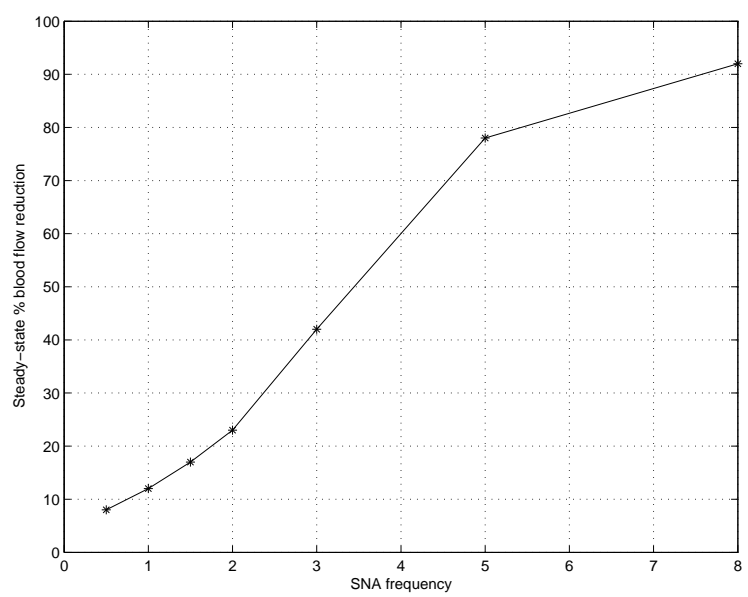

Fig. 5. Transformation for frequency stimulation

ward and feedback blocks are detailed in Table.1. Finally, the nonlinear feedback activation function is shown in Fig.6. A number of aspects of the model are noteworthy:

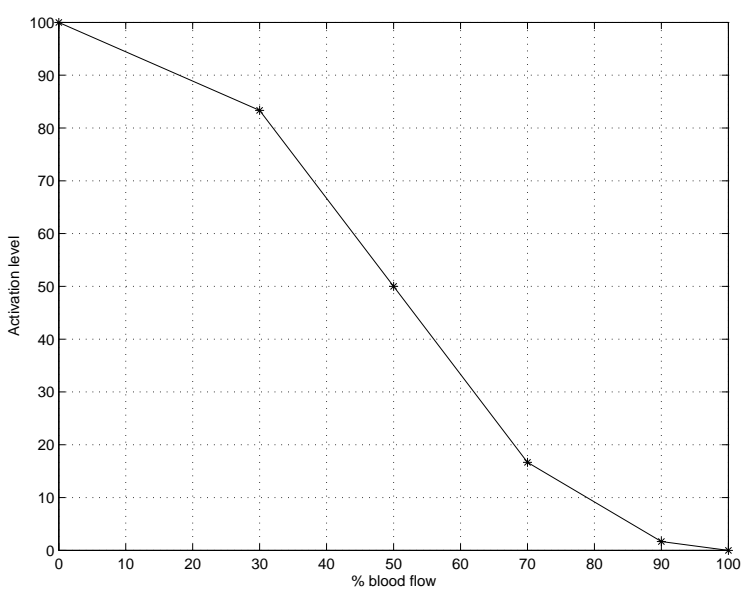

Fig. 6. Feedback activation function

- The feedback activation function has little effect below blood flow reduction levels of $30 \%$.

- The feedback branch has a potential activation level which is comparable with that of the feedforward section, when the blood flow level suffers any significant decrease (say to about $60 \%$, representing a reduction of $40 \%$ ). Note that the nonlinear feedback characteristic has been normalised approximately to the SNA stimulation level (of 100) at the summation block. Therefore the values of $k_{f}$ and $k_{b}$ can be directly compared.

- The feedback (vasodilatory) mechanism has a time response which is an order of magnitude greater than that of the feedforward response. This, in the main, accounts for the characteristic step response of the system.

The performance of the model in comparison to the recorded experimental data may now be evaluated by reference to, by way of example, Fig.7. Clearly, the model has captured the essence

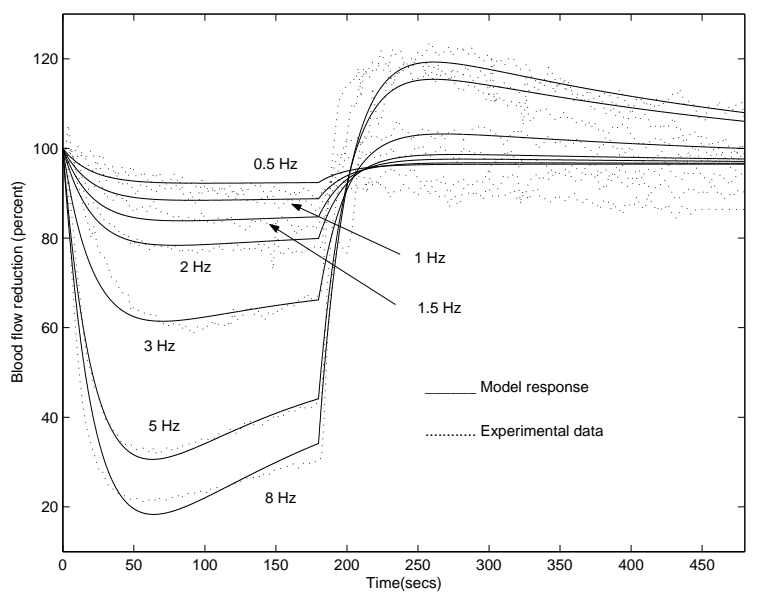

Fig. 7. Comparison of model response with experimental data

of the response contained in the the experimental data, though several features of the data fall outside the scope of the model, for example: 
- The data contains a significant amount of noise, as is to be expected with physiological experimental data. It is not the intention of the model to capture this. Arguably a better comparison could be made if the experimental data had been filtered prior to plotting, but the filter would introduce dynamics of its own which may interfere with the parameter determination.

- There is some longer term drift in the data, most noticable at lower levels of SNA activation. This is due to longer term autoregulatory effects, which are numerous. Conceivably, such an effect could be built into the model, but it is difficult (from an experimental point of view) to isolate such effects in order to develop components which will provide a generic description of these mechanisms.

The experimental responses in Fig.7, for higher activation levels (e.g. $3 \mathrm{~Hz}, 5 \mathrm{~Hz}$ and $8 \mathrm{~Hz}$ ) show a good pattern match to the 'template' response of Fig.4. At lower levels of SNA activation, the response is broadly (first-order) exponential, with little participation of the feedback loop.

\section{CONCLUSIONS}

This paper has developed a large-signal model for neurally-induced vasoaction in the renal vasculature. The model is based on physiological principles and the resulting intuitive relationship between the model parameters and its response has enabled the parameters to be determined on a trial-and-error basis. A Gauss-Newton gradient search algorithm was used in an effort to provide a more objective means of determining an optimum parameter set, but there was no improvement as a result of this, due to the difficulties with multiple local minima and the resulting sensitivity to initial parameter values. Some future work will examine the effectiveness of stochastic and concurrent search algorithms in providing an optimal parameter set.

The model presented in this paper focusses on the renal vasculature only and caution must be exercised in any attempt to extend its applicability to other vasculature components or, indeed, to $\mathrm{TPR}$ in general. It is known (see Section 1) that different vasculature beds may respond differently to similar vasoactive mechanisms, depending on the particular receptors (or mix of receptors) contained in the various vascular beds. Therefore, it is not reasonable to attempt to generalise this model to other vascular beds until it can be validated with appropriate experimental data. However, the renal vasculature is a major component of innervated resistance (approximately 30\%) and there- fore the model has a not insignificant relevance to TPR.

The model developed has deliberately separated dynamic and nonlinear elements (with a Hammerstein structure in the feedback path) in an effort to make the model as transparent as possible and facilitate parameter tuning. However, it is likely that an integrated model could provide a better fit to the data, though presenting a greater challenge in parameter determination (only via optimisation techniques).

We believe that this model can also be helpful as part of the modelling effort to investigate the origins of low-frequency (circa $0.1 \mathrm{~Hz}$ in humans) oscillations in blood pressure. Current models utilise a relatively simple linear first-order dynamic element to represent the resistance component of the vasculature (Ringwood and Malpas, 2001) and while this representation is adequate for small-signal situations, it is known that oscillations of a significant amplitude can occur under certain physiological conditions e.g. haemorrhage (Malpas and Burgess, 2000). Inclusion of the counteractive vasodilatory mechanism in the model presented in this paper is likely to make a significant change to predictions of oscillation amplitudes (particularly at higher amplitudes) compared to current models utilising simple linear models.

There is considerable scope for further work. In addition to the investigation of different optimisation techniques for model parameter determination, some improvements to the basic model structure are possible. In particular, Fig.7 confirms that, notwithstanding the employment of different time constants for feedforward and feedback components, the responses in positive-going and negative-going directions occur at different rates, with the slower response during the recovery from stimulation. This is perfectly reasonable, since:

- Recovery from SNA activation is passive and the smooth muscle may take longer to relax than contract under forced activation, and

- Hormones, paracrines, etc. may take much longer to disperse than the rate at which they were formed, since they rely on other aspects of the physiology to dilute them.

Finally, it is also anticipated that a model can be built which can combine the effects of both amplitude and frequency SNA stimulation. However, some careful analysis of the equivalence between these two mechanisms is necessary and there is an inherent difficulty in quantifying 'amplitude' stimulation on SNA fibres, since there is uncertainty as to the number of fibres covered by stimulating electrodes (and the mechanism by which 'amplitude' modulation occurs is via the recruitment of 
more/less fibres, 'amplitude' level on a single fibre being approximately fixed).

\section{REFERENCES}

Bellomo, R., J.A. Kellum, S.R. Wisniewski and M.R Pinsky (1999). Effects of norepinephrine on the renal vasculature in normal and endotoxemic dogs. Am. J. Respir. Crit. Care Med. 159, R1186-R1192.

Boulanger, C.M and P.M. Vanhoutte (1998). The endothelium: a modulator of cardiovascular health and disease. Dialogues in Cardiovascular Medicine 3, 4.

DiBona, G.F. and U.C. Kopp (1997). Neural control of renal function. Physiol. Rev 77, 75197.

Eppel, G.A., K.M. Denton, S.C. Malpas and R.G. Evans (2003). Nitric oxide in responses of regional kidney perfusion to renal nerve stimulation and renal ischaemia. Eur. J. Physiol 447, 205-213.

Guild, S.J., P.C. Austin, M. Navatikyan, J.V. Ringwood and S.C. Malpas (2001). Dynamic relationship between sympathetic nerve activity and renal blood flow: a frequency domain approach. Am. J. Physiol. (Regulatory Integrative Comp. Physiol.) 281, R206-R212.

Leonard, B.L., R.G. Evans, M.A. Navakatikyan and S.C. Malpas (2000). Differental neural cotrol of intrarenal blood flow. Am. J. Physiol. (Regul Ingr. Comp. Physiol) 297, R907R916.

Malpas, S.C. and D.E. Burgess (2000). Renal sna as the primary mediator of slow oscillations in blood pressure during haemorrhage. Am. J. Physiol. 279, R1299-R1306.

Navakatikyan, M.A., B.L. Leonard, R.G. Evans and S.C. Malpas (2000). Modeling the neural control of intrarenal blood flow. Clinical and Experimental Pharmacology and Physiology 27, 650652 .

Navar, L. Gabriel (1998). Integrating multiple paracrine regulators of renal microvascular dynamics. Am. J. Physiol. (Renal Physiol.) 274, F433 F444.

Richardson, D.R., D.C. Randall and D.F. Speck (1998). Cardiopulmonary System. 1st ed.. Fence Creek Publishing.

Ringwood, J.V. and S.C. Malpas (2001). Control of renal blood flow - the case for a nonlinear model. Am. J. Physiol. 280, R1105-R1115.

Sorensen, C.M., P.P. Leyssac, O. Skott and N.-H. Holstein-Rathlou (2000). Role of the reninangiotensin system in regulation and autoregulation of renal blood flow. Am. J. Physiol. (Regulatory Integrative Comp. Physiol.) 279, R1017-R1024. 